Supporting Information

\title{
Bacterial Vesicle-Cancer Cell Hybrid Membrane- Coated Nanoparticles for Tumor Specific Immune Activation and Photothermal Therapy
}

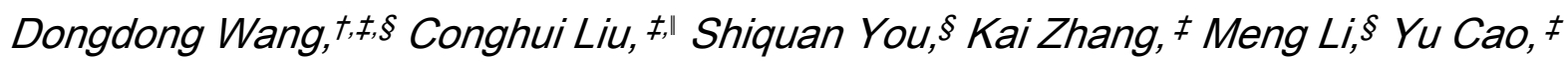
Changtao Wang, \& Haifeng Dong ${ }^{*,+, \neq, l}$ and Xueji Zhang ${ }^{* \neq,, 1}$

${ }^{\dagger}$ Research Center for Biomedical and Health Science, Anhui Science and Technology University, Fengyang, Anhui 233100, P. R. China

‡Beijing Key Laboratory for Bioengineering and Sensing Technology, Research Center for Bioengineering and Sensing Technology, School of Chemistry and Bioengineering,

University of Science \& Technology Beijing, Beijing 100083, P. R. China. 
§Beijing Key Lab of Plant Resource Research and Development, School of Science,

Beijing Technology and Business University, Beijing 100048, P. R. China.

ISchool of Biomedical Engineering, Health Science Center, Shenzhen University,

Shenzhen, Guangdong 518060, P. R. China

${ }^{*}$ Corresponding Authors:

Haifeng Dong (hfdong@ustb.edu.cn), Xueji Zhang (zhangxueji@ustb.edu.cn).

\section{EXPERIMENTAL SECTION}

Materials. Tetraethyl orthosilicate (TEOS), aqueous ammonia and absolute ethanol were obtained from Sinopharm Chemical Reagent Co., Ltd. (Beijing, China). Dopamine, Trihydroxymethyl aminomethane hydrochloride (Tris- $\mathrm{HCl}$ ), MTT and phenylmethanesulfonyl fluoride (PMSF) were from Sigma-Aldrich (St. Louis, MO, USA). Hoechst 33342 calcein-AM, PI, Dil and DiO were from Yeasen Biotech. Co., Ltd. (Shanghai, China). The bicinchoninic acid (BCA) protein assay kit and the membrane protein extraction kit were purchased from Beyotime Biotech. Co., Ltd. (Shanghai, China). All other reagents were of analytical grade. 
Instruments. The morphologies of NPs were measured by TEM (HT7700, Hitachi, Japan). The DLS were measured by Zetasizer Nano ZS90 system (Malvern, UK). The UV-visible absorption was detected using a UV-1800 spectrophotometer (Shimadzu, Japan). The cell fluorescence imaging experiments were carried out using CLSM (FV1200, Olympus, Japan). Ultracentrifuge (Beckman Coulter, Optima XPN-100, USA) was employed to centrifuge to collect OMVs. The cellular uptake and DC activation were measured by flow cytometry (Beckman Coulter, CytoFLEX, USA). The cellular uptake pathways of HPDA@[OMV-CC] NPs were measured by flow cytometry (ACAE NovoCyte, USA).A thermal imager (TiS65, Fluke, USA) was employed to record infrared thermal imaging. 

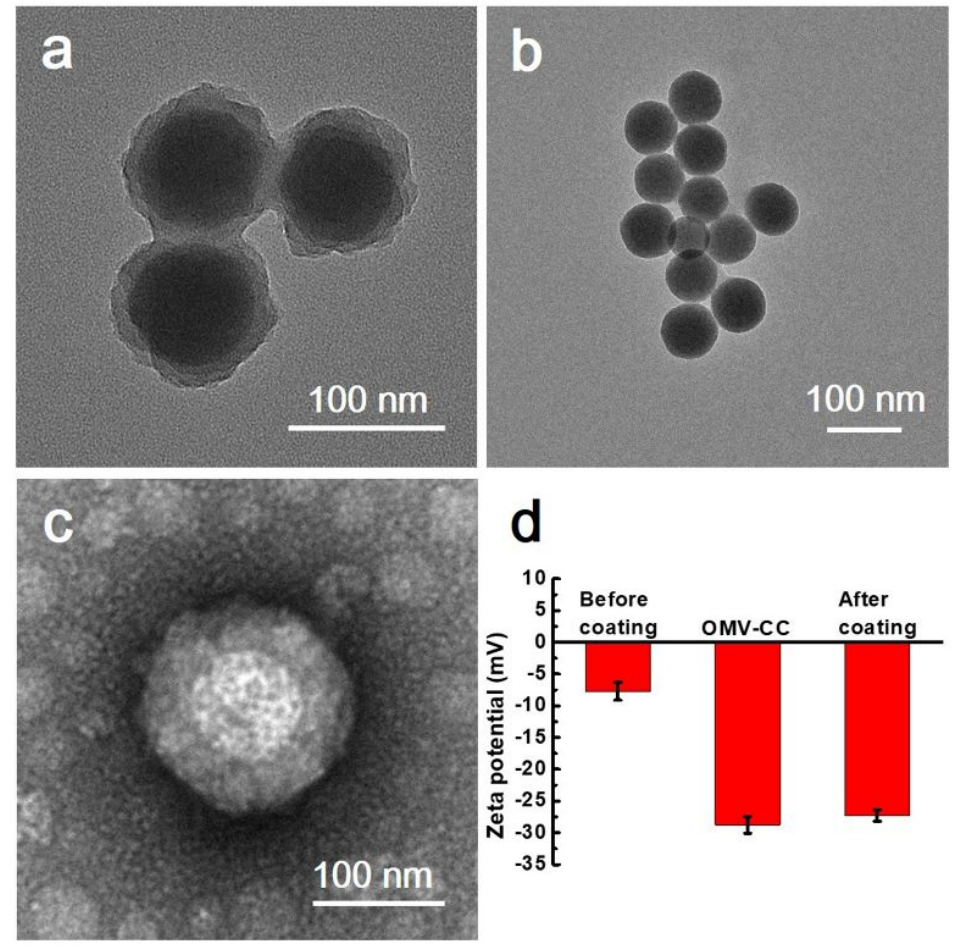

Figure S1. (a) TEM images of $\mathrm{SiO}_{2} @ P D A$ particles and (b) $\mathrm{SiO}_{2}$ particles. (c) TEM images of HPDA NPs negatively stained with uranyl acetate. (d) Surface zeta potential of HPDA, OMV-CC and HPDA@[OMV-CC] NPs.

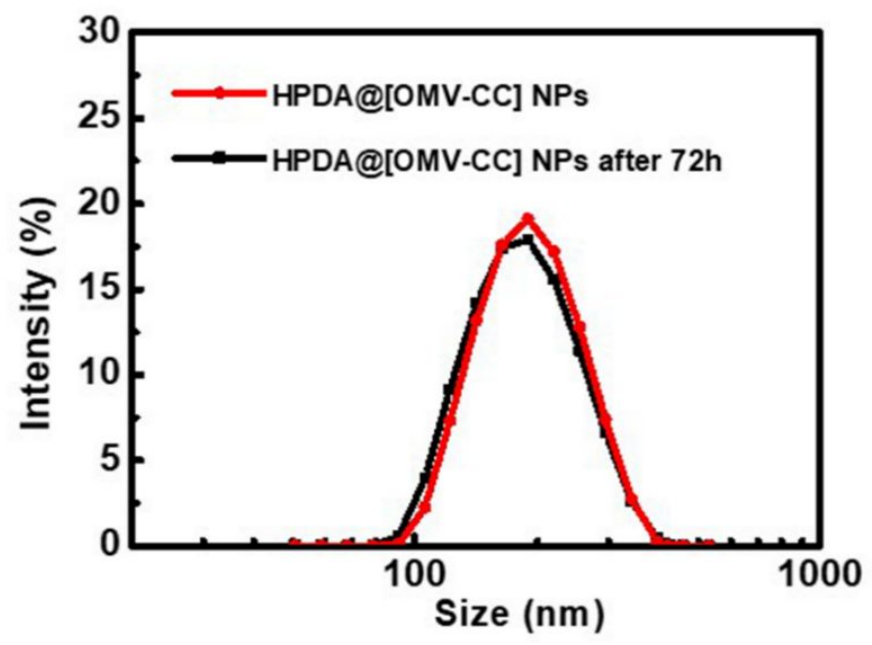


Figure S2. Hydrodynamic size of HPDA@[OMV-CC] NPs in serum and after storage for $72 \mathrm{~h}$ in serum.
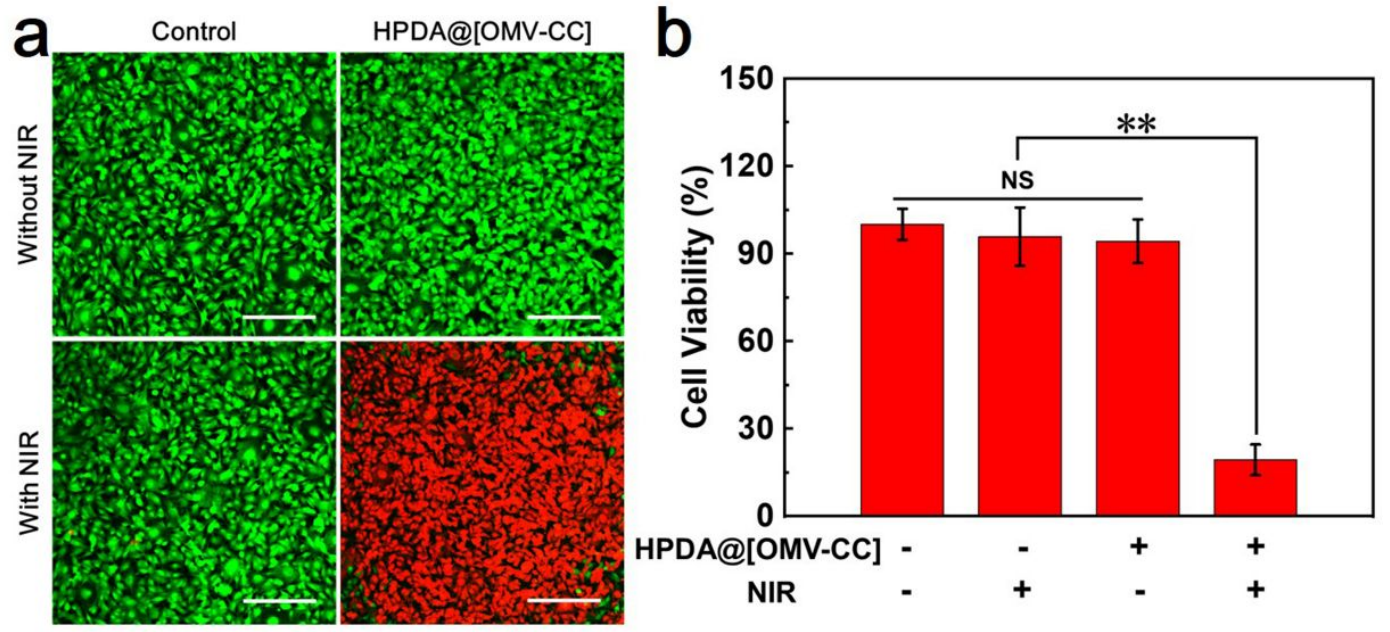

Figure S3. (a) Confocal fluorescent microscopy images of live/dead staining of B16-F10 cells cultured with HPDA@[OMV-CC] NPs without or with the NIR laser irradiation $\left(1064 \mathrm{~nm}, 1.0 \mathrm{~W} / \mathrm{cm}^{2}\right)($ scale bar $=200 \mu \mathrm{m}) .(\mathrm{b})$ The cell viabilities of B16F10 cells with different treatments. The data are shown as mean \pm SD $(n=3)$; NS indicates no statistical difference; and double asterisks indicate $\mathrm{P}<0.01$. 

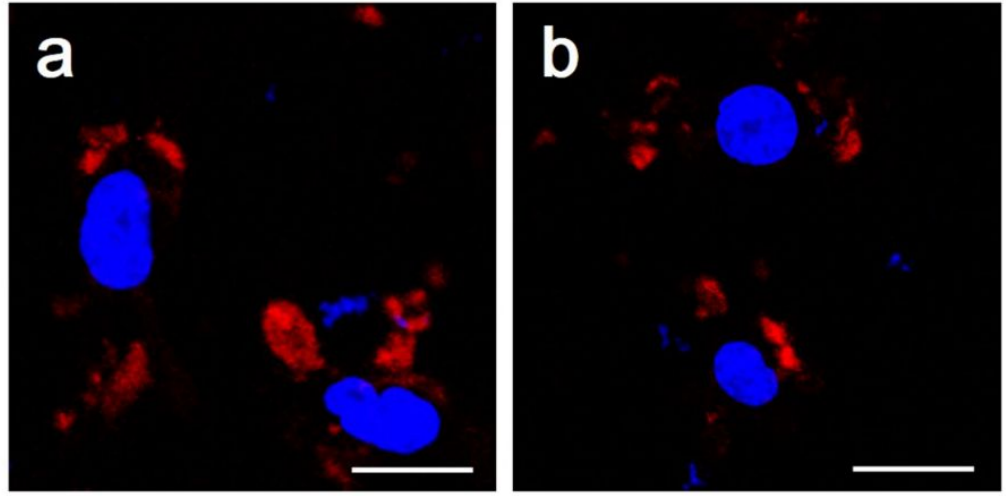

Figure S4. CLSM images of B16F10 cells stained with Hoechst 33342 and incubated with Dil-dyed HPDA@CC NPs (a) and HPDA@[OMV-CC] NPs (b) for 2h (scale bar = $20 \mu \mathrm{m})$.

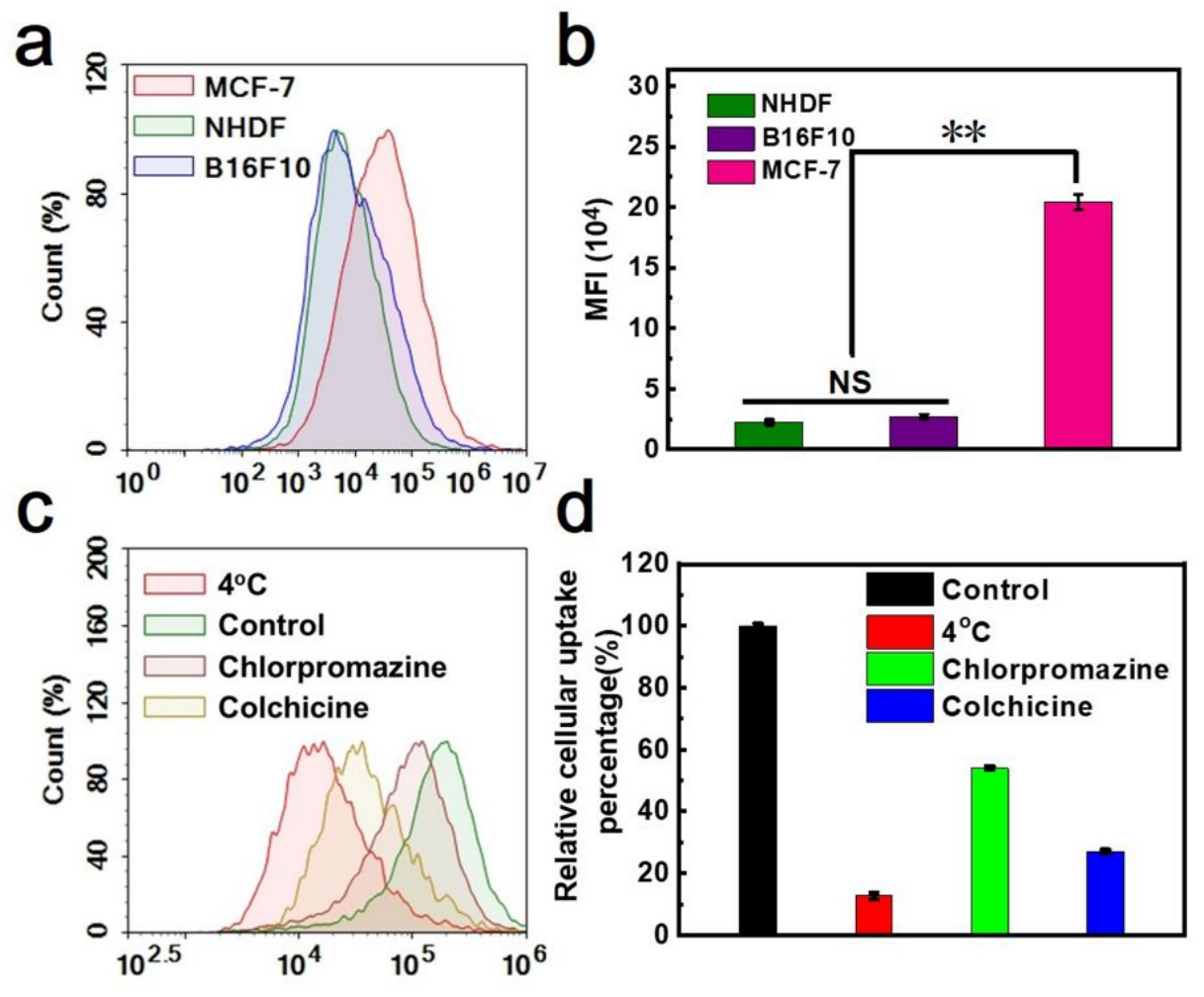


Figure S5. (a) Flow cytometric profiles and (b) mean fluorescence intensity of NHDF cells, MCF-7 cells and B16-F10 cells upon $4 \mathrm{~h}$ incubation with Dil-dyed HPDA@[OMVCC] NPs (CC membrane: MCF-7 cell membrane). (c) Flow cytometric profiles and (d) mean fluorescence intensity of cellular uptake pathways of HPDA@[OMV-CC] NPs. The data are shown as mean $\pm S D(n=3)$; NS indicates no statistical difference; and double asterisks indicate $\mathrm{P}<0.01$.

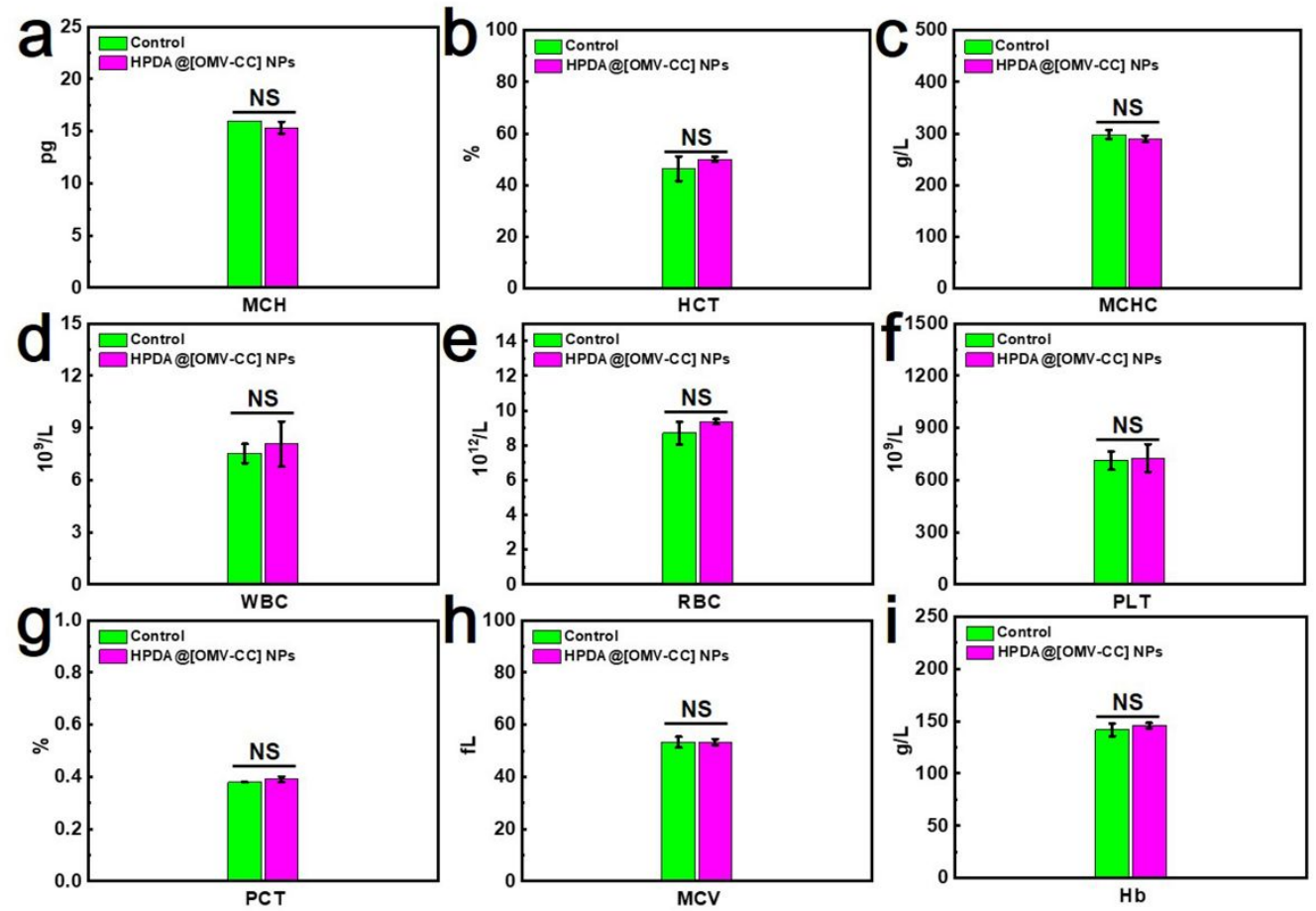

Figure S6. Blood levels of (a) mean corpuscular hemoglobin $(\mathrm{MCH})$, (b) hematocrit (HCT), (c) mean corpuscular hemoglobin concentration (MCHC), (d) white blood cells 
(WBC), (e) red blood cells (RBC), (f) platelets (PLT), (g) thrombocytocrit (PCT), (h) mean corpuscular volume (MCV), and (i) hemoglobin $(\mathrm{Hb})$. The data are shown as mean $\pm S D(n=5)$; NS indicates no statistical difference.

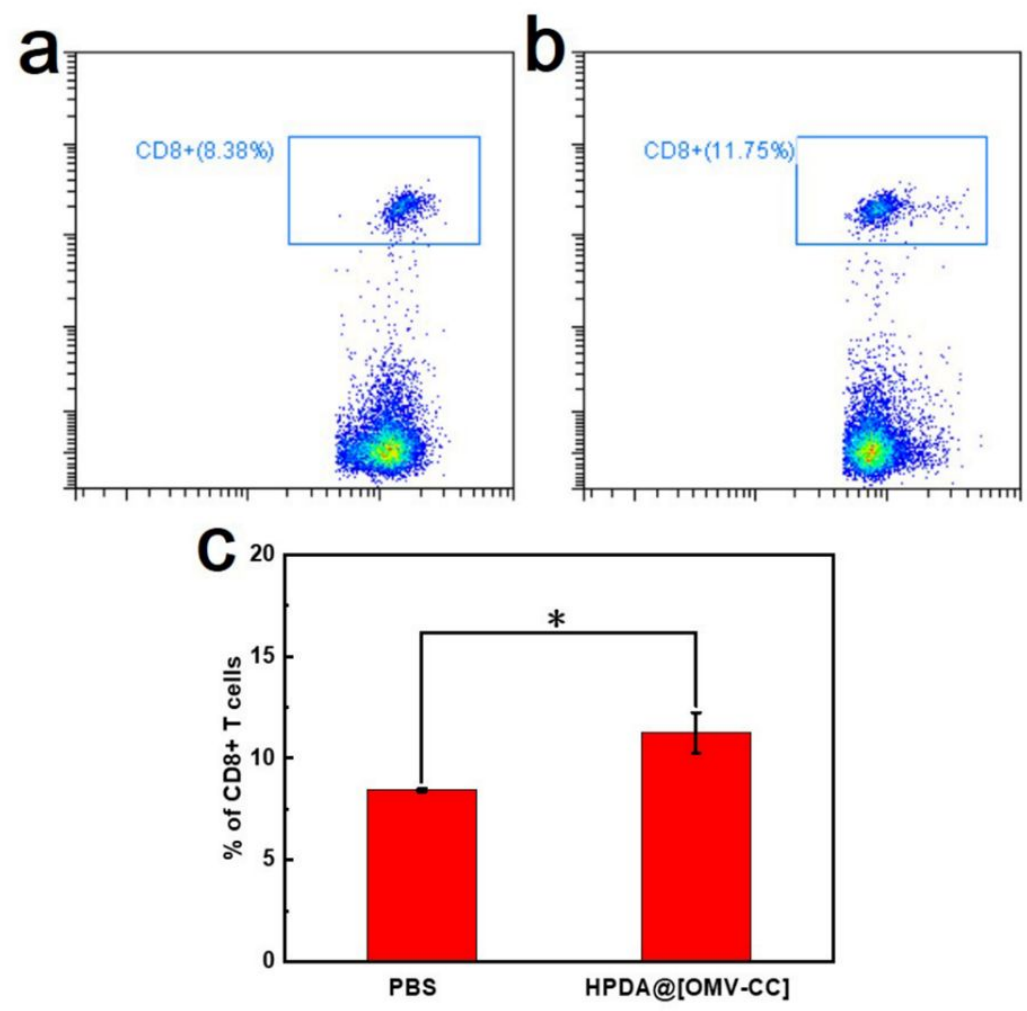

Figure S7. Flow cytometry analysis of the CD8 $+\mathrm{T}$ cells in spleen from (a) PBS treated group and (b) HPDA@[OMV-CC] NPs treated group. (c) Quantification of the percentage of CD8+ T cells in splenic monocytes corresponding to the data in (a) and (b). The data are shown as mean $\pm \mathrm{SD}(\mathrm{n}=5)$ and single asterisks indicate $\mathrm{P}<0.05$. 

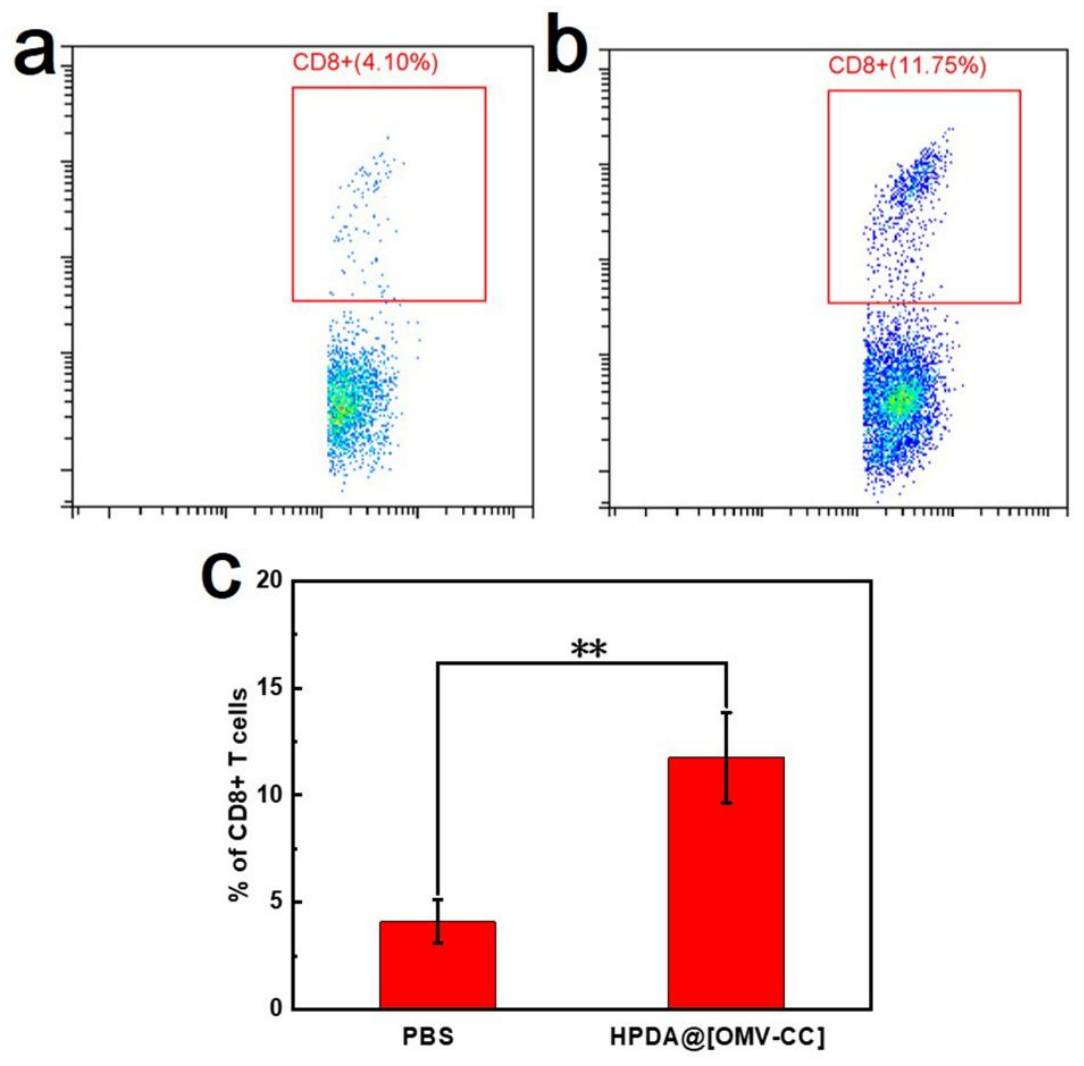

Figure S8. Flow cytometry analysis of the CD8+ T cells in tumor from (a) PBS treated group and (b) HPDA@[OMV-CC] NPs treated group. (c) Quantification of the percentage of CD8+ T cells in splenic monocytes corresponding to the data in (a) and (b). The data are shown as mean $\pm \mathrm{SD}(\mathrm{n}=5)$; double asterisks indicate $\mathrm{P}<0.01$. 


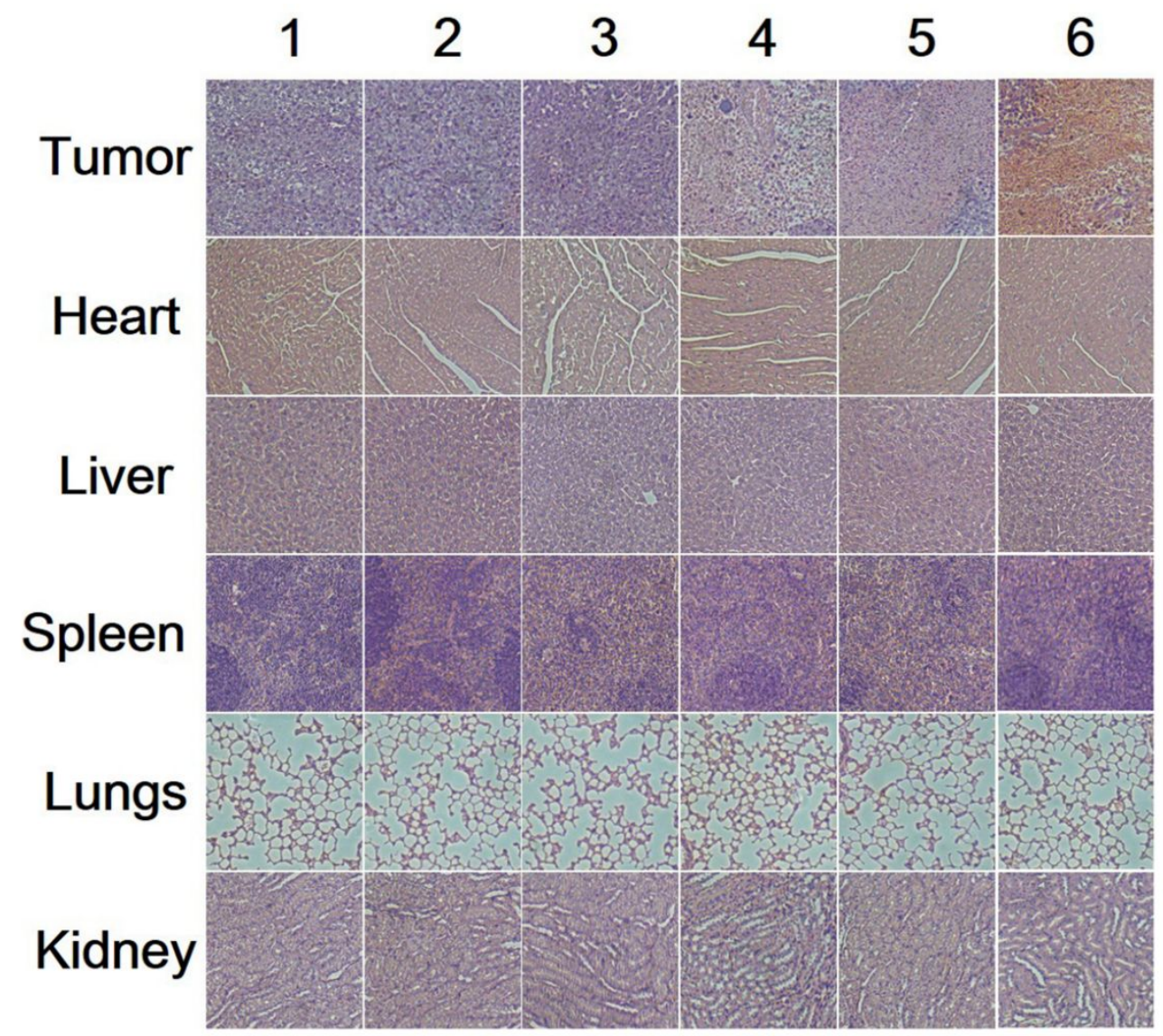

Figure S9. H\&E staining of the major organs (heart, liver, spleen, lung, and kidney) after mice receiving different treatments for 13 days (1: PBS, 2: NIR laser, 3: HPDA@CC NPs, 4: HPDA@[OMV-CC] NPs, 5: HPDA@CC NPs with NIR laser, 6: HPDA@[OMVCC] NPs with NIR laser). 